We try to publish authors' responses in the same edition with readers' comments. Time constraints might prevent this in some cases. The problem is compounded in a bimonthly journal where continuity of comment and redress are difficult to achieve. When the redress appears 2 months after the comment, 4 months will have passed since the original article was published. Therefore, we would suggest to our readers that their correspondence about published papers be submitted as soon as possible after the article appears.

\section{Treatment of Otitis Externa}

To the Editor: I would like to expand on two points made in Halpern et al's recent article about otitis externa treatment patterns. ${ }^{1}$ Physician behaviors seem to vary in actual practice from what is accepted practice. ${ }^{2}$ I am certain that many readers reviewed these results with a thoughtful reevaluation of their own practice habits.

In describing a high rate of systemic antibiotic usage in treating otitis externa, the author did mention a comorbid condition that would justify aggressive use of antipseudomonal antimicrobials. Malignant external otitis is an important concern in elderly diabetic patients with external otitis. ${ }^{3}$ This uncommon but serious condition can be life-threatening, causing erosion and osteomyelitis of the skull base, which can lead to meningitis, brain abscess, and even death. Progressive cranial nerve palsies have also been reported. Family physicians must be aware of this association and begin treatment urgently with appropriate consultation for careful surgical debridement and management.

The second point of interest relates to the lack of "meticulous cleaning of the external auditory meatus" performed by the study group of physicians; of physicians studied, only 7 percent of adults and 2 percent of children were treated with this procedure. The authors mention the lack of documented efficacy of external auditory canal irrigation in the literature as a theoretical justification for not performing removal of discharge and debris. Additional morbidity has been associated with external auditory canal irrigation, however, resulting in reported malignant external otitis in an elderly diabetic patient. ${ }^{4}$

Further investigation of the efficacy of external auditory canal irrigation, comparing outcomes with those of a control group, appears to be indicated if this procedure is to be recommended in future clinical guidelines.

Paul Evans, DO

College of Osteopathic Medicine

Oklahoma State University, Tulsa

\section{References}

1. Halpern MT, Palmer CS, Seidlin M. Treatment patterns for otitis externa. J Am Board Fam Pract 1999;12:17.
2. Evans P. Otitis externa. In: Taylor RB editor. Manual of family practice. Boston: Lippincott-Raven, 1996.

3. Evans P, Hofmann LA. Malignant external otitis - a case report and review. Am Fam Physician 1994;49:427-31.

4. Ford GR, Courteney-Harris RG. Another hazard of ear syringing: malignant external otitis. J Laryngol Otol 1990:104; 70910.

The above letter was referred to the authors of the article question, who offer the following reply.

To the Editor: We would like to thank Dr. Evans for his thoughtful comments on our recent manuscript. As Dr. Evans indicates, malignant otitis externa is a potentially life-threatening condition. As the main risk group for this condition is elderly patients with diabetes, we agree that aggressive treatment of otitis externa in this population with oral or intravenous antibiotics is very appropriate. As Dr. Evans further points out, external auditory canal irrigation should also be used cautiously in this population.

Malignant external otitis media. however, is a very rare condition. The overwhelming majority of patients who have with external otitis media do not fall into the specified high-risk group for the malignant condition. Thus, the high level of treatment of otitis externa with oral as well as topical medications observed in our study (at more than 40 percent of the physician visits) is not only unnecessary but also increases costs and the likelihood of side effects.

$$
\begin{array}{r}
\text { Michael T. Halpern, MD, PhD } \\
\text { Cynthia S. Palmer, MS } \\
\text { MEDTAP International } \\
\text { Mindell Seidlin, MD } \\
\text { Daiichi Pharmaceutical Corporation }
\end{array}
$$

\section{Reimbursement for Flexible Sigmoidoscopy}

To the Editor: Thanks to Dr. Mulvey ${ }^{1}$ for the opportunity to provide additional information and comments regarding the health care system, which discriminates against family physicians who wish to provide diagnostic and therapeutic services such as flexible sigmoidoscopy, endoscopic biopsy, and colonoscopy. His letter accurately reflects yet another dimension of this problem. I have further referenced the consequences of regulatory unfairness in the area of reimbursement for flexible sigmoidoscopy. ${ }^{2}$

The question remains regarding the political will of physicians within the medical specialty of family practice. Certainly the American Academy of Family Physicians has been a source of support for family physicians who wish to maintain the right to incorporate emerging diagnostic and therapeutic skills into their practice. The Residency Review Committee through their accreditation process attempts to maintain the breadth of the specialty despite a variety of 